\title{
A Detailed Questionnaire for the Evaluation of Health Management in Dairy Sheep and Goats
}

\author{
Daphne T. Lianou ${ }^{1}$, Ioanna P. Chatziprodromidou ${ }^{2}$, Natalia G. C. Vasileiou ${ }^{3}$, \\ Charalambia K. Michael ${ }^{1}$, Vasia S. Mavrogianni ${ }^{1}$, Antonis P. Politis ${ }^{1}$, Nikos G. Kordalis ${ }^{1}$, \\ Charalambos Billinis ${ }^{1}$, Alexios Giannakopoulos ${ }^{1}$, Elias Papadopoulos 4 , Ilias Giannenas ${ }^{4}$, \\ Katerina S. Ioannidi ${ }^{1}$, Angeliki I. Katsafadou ${ }^{1}$, Dimitris A. Gougoulis ${ }^{1}$, Delia Lacasta ${ }^{5}$, \\ Mariangela Caroprese ${ }^{6}$ and George C. Fthenakis ${ }^{1, * \mathbb{D}}$ \\ 1 Veterinary Faculty, University of Thessaly, 43100 Karditsa, Greece; dlianou@vet.uth.gr (D.T.L.); \\ cmichail@uth.gr (C.K.M.); vmavrog@vet.uth.gr (V.S.M.); apolitis.vet@gmail.com (A.P.P.); \\ nikolaoskordalis@gmail.com (N.G.K.); billinis@vet.uth.gr (C.B.); alexiosg@yahoo.gr (A.G.); \\ kate_ioan@windowslive.com (K.S.I.); agkatsaf@vet.uth.gr (A.I.K.); dgoug@vet.uth.gr (D.A.G.) \\ 2 Medical School, University of Patras, 26504 Patras, Greece; ioannachatzi@med.upatras.gr \\ 3 Faculty of Animal Science, University of Thessaly, 41500 Larissa, Greece; vasileiounat@gmail.com \\ 4 Faculty of Veterinary Medicine, Aristotle University of Thessaloniki, 54124 Thessaloniki, Greece; \\ eliaspap@vet.auth.gr (E.P.); igiannenas@vet.auth.gr (I.G.) \\ 5 Animal Pathology Department, Instituto Agroalimentario de Aragón-IA2 Universidad de Zaragoza-CITA, \\ Veterinary Faculty of Zaragoza, 50013 Zaragoza, Spain; dlacasta@unizar.es \\ 6 Department of Sciences of Agriculture, Food and Environment (SAFE), University of Foggia, 71122 Foggia, \\ Italy; mariangela.caroprese@unifg.it \\ * Correspondence: gcf@vet.uth.gr
}

Received: 7 August 2020; Accepted: 21 August 2020; Published: 24 August 2020

Simple Summary: The objective of this work was to develop, use and present a detailed questionnaire for the evaluation of health management in dairy small ruminants. The questionnaire includes 442 questions, which cover seven sections (general, infrastructure, animals, production characteristics, health management, nutrition, human resources). The average duration of the interview for completion of the questionnaire was $64 \mathrm{~min}$. The questionnaire can be used for research work in the field, to record details in the farms into the study. In accord with the needs of a particular study, the questionnaire can be extended, by adding therein more specific questions or omitting some deemed to be less important. Moreover, the questionnaire can also be used for routine monitoring purposes, as a useful means to record and maintain details of farms during clinical work.

Abstract: The objective of this work was to develop, use and present a detailed questionnaire for the evaluation of health management in dairy small ruminants; it includes 442 questions organised in seven sections: general, infrastructure, animals, production characteristics, health management, nutrition, human resources. Consistency of replies was evaluated in 27 farmers, interviewed twice. Inconsistent replies were given by all farmers to 30 different questions (Cronbach's coefficient alpha: 0.987). Then, interviews were performed in 444 farms around Greece. Mean duration of an interview was $63.6 \mathrm{~min}$. Clarifications were requested by 273 farmers to 22 different questions (maximum per farmer: 8). The experience of the investigator, the primary language of farmers and asking clarifications by the farmers affected the duration of the interview. The questionnaire can be used for research work in the field, to record details in the farms under study. In accord with the needs of a particular study, it can be modified, by adding more specific questions or omitting others deemed of less importance. Moreover, it can also be used for routine monitoring purposes, as a useful means to record and maintain details of farms during clinical work. To the best of our knowledge, the questionnaire is the most extensive and detailed one available internationally for dairy small ruminants. 
Keywords: epidemiology; field study; health management; infrastructure; nutrition; production characteristics; questionnaire; survey

\section{Introduction}

Research work can be greatly facilitated by using standardised questionnaires to gather information. These can be applied in varying situations (e.g., farms in survey studies), contributing to producing comparable results, which would further allow evaluation of findings between studies. Moreover, in animal population medicine, questionnaires are important for the detailed assessment of farms (e.g., management system, infrastructure, feeding regime, production outcomes, animal populations etc.), the outcome of which can guide the subsequent efficient usage of resources (e.g., ancillary tests to be performed). To the best of our knowledge, no detailed questionnaire is available in the international literature for use in small ruminants. The objective of this work was to develop, use and present a detailed questionnaire for the evaluation of health management in dairy sheep flocks/goat herds.

\section{Materials and Methods}

\subsection{The Questionnaire}

The questionnaire includes 442 questions organised in seven sections. The full questionnaire is presented in Table S1.

\subsection{Development}

The questionnaire was developed at the Department of Obstetrics and Reproduction of the Veterinary Faculty of the University of Thessaly; the Department is also a training centre of the European College of Small Ruminant Health Management and, in past years, has performed many field projects around Greece, from which experience was drawn to set up and develop a detailed questionnaire. Members of the academic staff of the Department and field veterinarians have contributed in the development of the questionnaire. The protocols of the study were approved by the academic board of the Faculty, meeting 34/03.04.19. Further contributions were made by the academic staff of the Department of Microbiology and Parasitology of the Faculty in relation to issues of biosecurity and wildlife, as well as by academic staff of the Laboratory of Parasitology and the Laboratory of Nutrition of the Faculty of Veterinary Medicine of the Aristotle University of Thessaloniki in relation to issues of antiparasitic drugs and nutritional management, respectively. The questionnaire was originally prepared in the Greek language.

After completion of the final draft of the questionnaire, a pilot test was conducted; seven known farmers (i.e., with whom there had been a previous professional collaboration) and four unknown farmers (i.e., ones whom the authors had never met before) were interviewed, in order to better understand whether they would comprehend the questions and to evaluate the flow of the interview. After taking into account the responses and the reactions of these 11 farmers, appropriate modifications were made and the questionnaire was finalised. There were seven sections in the questionnaire, which included open, multiple-choice, dichotomous and scaling questions (Table 1).

After finalising the questionnaire, 27 farmers (specifically, the persons within the owning families of each farm, who managed and followed up the business activities and operations in the respective sheep flocks/goat herds) were interviewed, with the objective to test consistency of the replies of the farmers. These farmers were visited and interviewed twice, with an interval of two to three months in-between, in both occasions by the same investigator (DTL). Then, the answers received in the two interviews were compared. Five of the questions (no. 007: Geographical coordinates; 042, 053, 067: Dimensions of structures; 046: Orientation of the building) were not asked, because relevant data 
were to be collected using hand-held global positioning system Garmin units or a laser measuring tool as appropriate. Another four of the questions (001: Farm reference; 005: Date of the visit; 006: Veterinarian(s); 033: Availability of access road) also were not asked, because the answers were known before the start of the interview. Additionally to the interview, as part of a thorough evaluation of the site, another investigator (CKM), also present during each visit, recorded details regarding as many as possible of 48 questions (Appendix A), whilst the breeds of the sheep/goats in the farm (questions: 137-140) were confirmed by a European Veterinary Specialist in Small Ruminant Health Management (GCF).

Table 1. Types of questions (n) in the questionnaire according to the various sections therein.

\begin{tabular}{cccccc}
\hline & \multicolumn{5}{c}{ Type of Question } \\
\hline Section & Open & Multiple-Choice & Dichotomous & Scaling & Total \\
\hline General & 8 & 2 & 1 & 0 & 11 \\
Infrastructure & 39 & 17 & 63 & 0 & 119 \\
Animals & 43 & 4 & 38 & 0 & 85 \\
Production characteristics & 15 & 2 & 2 & 0 & 19 \\
Health management & 61 & 16 & 12 & 3 & 150 \\
Nutrition & 12 & 13 & 8 & 0 & 37 \\
Human resources & 12 & 1 & 194 & 3 & 442 \\
\hline Total & 190 & 55 & & 0 & 21 \\
\hline
\end{tabular}

\subsection{Application}

In the final stage, 444 farmers (specifically, the persons within the owning families of each farm, who managed and followed up the business activities and operations in the respective sheep flocks/goat herds) were interviewed: 325 sheep flocks and 119 goat herds in all the 13 administrative regions of Greece (Appendix B) were visited, in order to interview the respective farmers. Veterinarians active in small ruminant health management around Greece, were contacted by telephone and asked if they wished to collaborate in the investigation. In total, 48 veterinarians were contacted; of these, 47 (97.9\%) agreed to collaborate. Farms were selected by the collaborating veterinarians on convenience basis (willingness of farmers to accept a visit by University personnel for an interview). Each of these veterinarians had a stable, although not contractual, association with the respective farm, among those selected for visit, and were responsible for their decisions and actions in relation to the health and welfare of the animals therein, in full accord with the relevant veterinary conduct codes [1,2]. The investigators visited all farms for the interviews. Of the 47 veterinarians, 45 accompanied the investigators during the interviews. Visits were scheduled to 446 farms, but on two occasions ( $0.4 \%)$, whilst the investigators had already arrived at these farms, the respective farmers mentioned that they did not want to take the interview and refused to collaborate. Details of the farms are in Table 2 and their locations round the country are shown in Figure 1.

Table 2. Details of farms, in which interviews were performed.

\begin{tabular}{cccccc}
\hline \multirow{2}{*}{ Animal Species in the Farms } & \multicolumn{3}{c}{ Management System $^{\mathbf{1}}$} \\
\cline { 2 - 6 } & Intensive & Semi-Intensive & Semi-Extensive & Extensive & Total \\
\hline Sheep & 43 & 151 & 107 & 24 \\
Goats & 9 & 31 & 58 & 21 & 119 \\
\hline Total & 52 & 182 & 165 & 45 \\
\hline
\end{tabular}

\footnotetext{
${ }^{1}$ Management system practiced in the farms was defined according to the European Food Safety Authority [3].
}

All the interviews were performed by the same investigator (DTL). The duration of the interview was recorded. Any questions for which clarifications were requested by the respondents, were recorded. 
The spontaneous comments of the farmers during the interview were also recorded by the interviewing investigator (DTL), whilst the spontaneous comments of the veterinarians were recorded by another investigator (GCF).

Details regarding as many as possible of 48 questions (Appendix A) were also recorded during the on-site visit by another investigator (CKM), who was present in the farms. The breeds of the sheep/goats in the farm (questions: 137-140) were confirmed by a European Veterinary Specialist in Small Ruminant Health Management (GCF). After conclusion of the interview, details regarding as many as possible of 56 questions (Appendix C) were also collected by an investigator (DTL) from the veterinarian, who had arranged the visit.

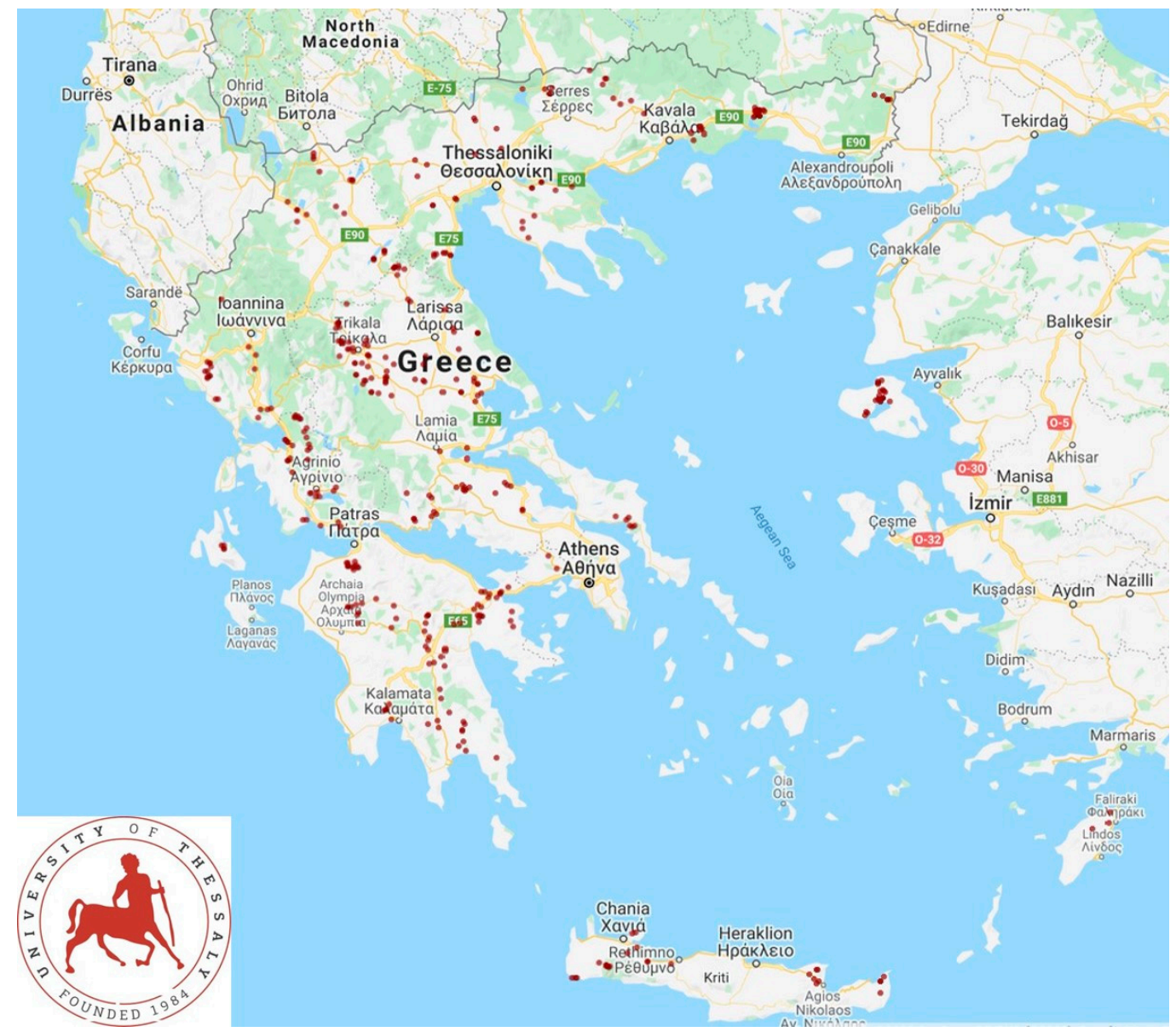

Figure 1. Location of 444 sheep flocks/goat herds around Greece, in which interviews were performed.

\subsection{Data Management and Analysis}

During the development of the questionnaire, an inconsistent reply in the interviews by the two investigators was considered when the answers of a farmer to the same question differed between the two interviews; for open questions that required a quantitative answer (e.g., annual milk production in the farm, average weight of lambs at slaughter, total amount of concentrates purchased) only differences of over $5 \%$ between the two replies were taken into account. During the application of the questionnaire, the duration of the interview was calculated from the time the first question was asked to the time the final question was answered; durations were rounded to the nearest minute.

The data were entered into Microsoft Excel. Basic descriptive analysis was initially performed.

Analysis of variance or analysis of correlation, as appropriate, was performed between the number of inconsistent replies by a farmer and the animal species farmed, the management system practiced, their sex, age, farming experience, education and whether they were in full-time farming. Then, 
the frequency of inconsistent replies among the various types of questions (open, multiple choice, dichotomous, scaling) was evaluated in a table of cross-categorised frequency data by use of the Pearson chi-square test. The internal consistency of the answers in the repeated interviews of the 27 farmers was evaluated by calculating Cronbach's coefficient alpha [4] in a statistical software package [5].

Differences in the duration of the interview and in the number of clarifications asked between farmers according to animal species farmed, management system practiced, sex, age, farming experience, education and whether they were full-time farmers or not, as well as the administrative region of the country, were evaluated by means of analysis of variance or analysis of correlation, as appropriate.

Linear regression of the duration of the interviews and the number of clarifications throughout the study was assessed. Also, the duration of the interview and the clarifications asked by farmers were calculated separately for the initial 222 interviews and for the last 222 interviews; then, comparisons were made by analysis of variance between the two values for each of these two measures.

In all cases, level of significance was set at $p \leq 0.05$.

\section{Results}

\subsection{Confirmation of the Questionnaire}

Inconsistencies were found in the replies of all 27 (100\%) farmers in the two interviews. Among all farmers, the median value of inconsistent replies was $6(1.4 \%)$ per farmer (min-max $=3-10 ; 0.7 \%-2.3 \%$ ). There was no association between the number of inconsistent replies by a farmer and the animal species farmed, the management system practiced, their sex, age, farming experience or education, as well as whether they were in full-time farming or not ( $p>0.11$ for all comparisons).

Inconsistencies were found in the replies to 30 different questions in the questionnaire $(6.9 \%)$. The median value of inconsistent replies was $5(1.2 \%)$ (min-max $1-14 ; 0.2 \%-3.2 \%)$ when only questions with inconsistent replies $(n=30)$ were taken into account (Appendix D). Most of these questions (76.7\%) were open type questions; the proportion of inconsistent replies among open questions (12.7\%) was significantly higher than that among all other types of questions $(2.8 \%)(p<0.001)$.

Cronbach's coefficient alpha was found to be 0.987 .

\subsection{Application of the Questionnaire}

\subsubsection{Duration of the Interview}

The mean value ( \pm standard error of the mean) of the duration of the interview was $63.6 \pm 0.3 \mathrm{~min}$. There was evidence for a significant difference in the duration of the interview between farmers who spoke $(n=418)$ or did not speak $(n=26)$ Greek as their primary language: $63.6 \pm 0.3$ versus $66.0 \pm 0.2 \mathrm{~min}(p=0.035)$. There were no differences in the duration of the interview between (a) sheep or goat farmers $(63.6 \pm 0.3$ or $64.0 \pm 0.5 \mathrm{~min}$, respectively; $p=0.50),(\mathrm{b})$ farmers practicing intensive, semi-intensive, semi-extensive or extensive management system $(62.7 \pm 0.8,63.7 \pm 0.4,64.2 \pm 0.5$ or $63.6 \pm 0.9 \mathrm{~min}$, respectively; $p=0.467),(\mathrm{c})$ male $(n=414)$ or female $(n=30)$ farmers $(63.7 \pm 0.3$ or $64.6 \pm 0.3 \mathrm{~min}$, respectively; $p=0.38)$, (d) farmers with primary $(n=144)$, secondary $(n=171)$, vocational $(n=69)$, tertiary technological $(n=18)$ or tertiary university $(n=42)$ education $(63.8 \pm 0.3,64.1 \pm 0.3$, $64.0 \pm 0.3,61.8 \pm 0.3$ or $62.6 \pm 0.3 \mathrm{~min}$, respectively; $p=0.32)$, (e) full-time $(n=397)$ or part-time $(n=47)$ farmers $(63.7 \pm 0.3$ or $63.5 \pm 0.3 \mathrm{~min}$, respectively; $p=0.82)$ and $(\mathrm{f})$ farmers who employed $(n=157)$ or did not employ $(n=287)$ staff $(63.3 \pm 0.3$ or $64.0 \pm 0.3 \mathrm{~min}$, respectively; $p=0.19)$. Moreover, there was no association between duration of the interview and age $(r=-0.014)$ or experience $(r=-0.034)$ of farmers ( $p=0.38$ or 0.23 , respectively), nor between duration of the interview and the time spent by the farmer working at the farm $(\mathrm{r}=0.013 ; p=0.40)$. There was also no significant difference in the duration of the interview between farmers in the 13 administrative regions of the country $(p=0.28)$.

The duration of the interviews decreased progressively, as the investigation advanced: slope of the duration throughout the study was -0.0075 (standard error: 5.5877) $(p=0.0002)$ (Figure 2). 
The mean duration of the interview was longer during the initial 222 than during the last 222 interviews: $64.8 \pm 0.3$ versus $62.7 \pm 0.3 \mathrm{~min}(p=0.0001)$. This was evident among farmers who spoke Greek as their primary language: $64.6 \pm 0.3$ versus $62.7 \pm 0.3 \mathrm{~min}(p=0.0007)$, but not among ones who did not: $66.5 \pm 0.2$ versus $63.0 \pm 0.1 \mathrm{~min}(p=0.066)$.

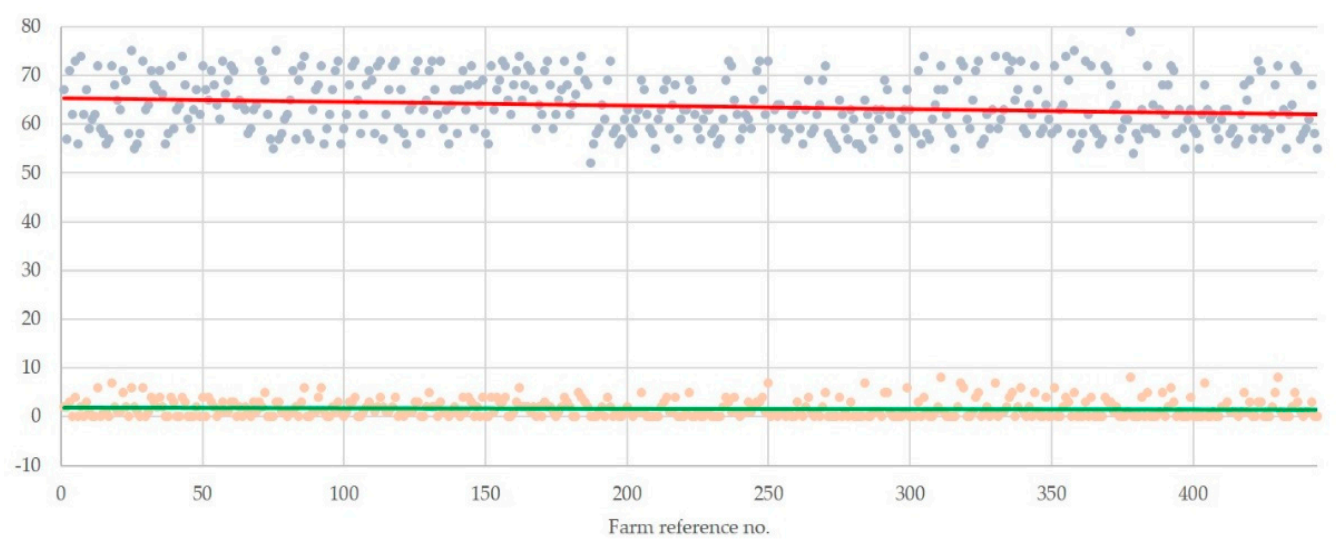

Figure 2. Plot diagram of the progressive duration of the interviews (fade blue spots) and clarifications asked by the farmers (fade orange spots), with slope lines: solid red line (slope: -0.0075 ) and solid green line (slope: -0.0006$)$, respectively $(n=444)$ (the vertical axis shows durations of interviews ( $\mathrm{min}$ ) and no. of clarifications asked by the farmers).

\subsubsection{Clarifications Asked by the Farmers}

Clarifications were asked by $273(61.5 \%)$ farmers in total (median value: one question per farmer; $0.2 \%$ ) (Appendix E). There was no association between the number of clarifications asked and (a) sheep or goat farmers $(1.5 \pm 0.1$ or $1.8 \pm 0.2$ questions, respectively; $p=0.14)$, (b) farmers practicing intensive, semi-intensive, semi-extensive or extensive management system $(1.3 \pm 0.2,1.6 \pm 0.1,1.7 \pm 0.1$ or $1.6 \pm 0.3$ questions, respectively; $p=0.74)$, (c) male or female farmers $(1.6 \pm 0.1$ or $2.0 \pm 0.1$ questions, respectively; $p=0.27$ ), (d) farmers with primary, secondary, vocational, tertiary technological or tertiary university education $(1.5 \pm 0.1,1.7 \pm 0.1,1.5 \pm 0.1,1.6 \pm 0.1$ or $1.6 \pm 0.1$ questions, respectively; $p=0.94)$, (e) full-time or part-time farmers (1.6 \pm 0.1 or $1.8 \pm 0.1$ questions, respectively; $p=0.54)$ and (f) primary language of the farmers ( $1.6 \pm 0.1$ or $1.7 \pm 0.1$ questions, respectively; $p=0.90)$. Moreover, there was no association between the number of clarifications asked and age $(r=-0.001)$ or experience $(r=-0.036)$ of the farmers ( $p=0.49$ or 0.22 , respectively), nor between the number of clarifications asked and the time spent by the farmer working at the farm $(r=0.019 ; p=0.35)$. Finally, there was also no significant difference in the number of clarifications asked between farmers in the 13 administrative regions of the country $(p=0.85)$.

The number of clarifications asked by the farmers progressively did not change: slope of the clarifications asked throughout the study was -0.0006 (standard error: 1.8402) $(p=0.18)$ (Figure 2). The mean number of clarifications asked by the farmers did not differ significantly between the initial and the last 222 interviews: $1.7 \pm 0.1$ versus $1.5 \pm 0.1(p=0.34)$.

Clarifications were asked in 22 different questions (5.0\%) (Appendix E). Most of these questions $(81.8 \%)$ were dichotomous questions; the proportion of questions for which clarifications were requested among dichotomous questions $(9.3 \%)$ was significantly higher than that among all other types of questions $(1.7 \%)(p<0.001)$.

There was a clear correlation between the number of questions for which clarifications were asked by a farmer, and the respective duration of the interview $(r=0.76 ; p<0.0001)$ (Figure 3$)$. 


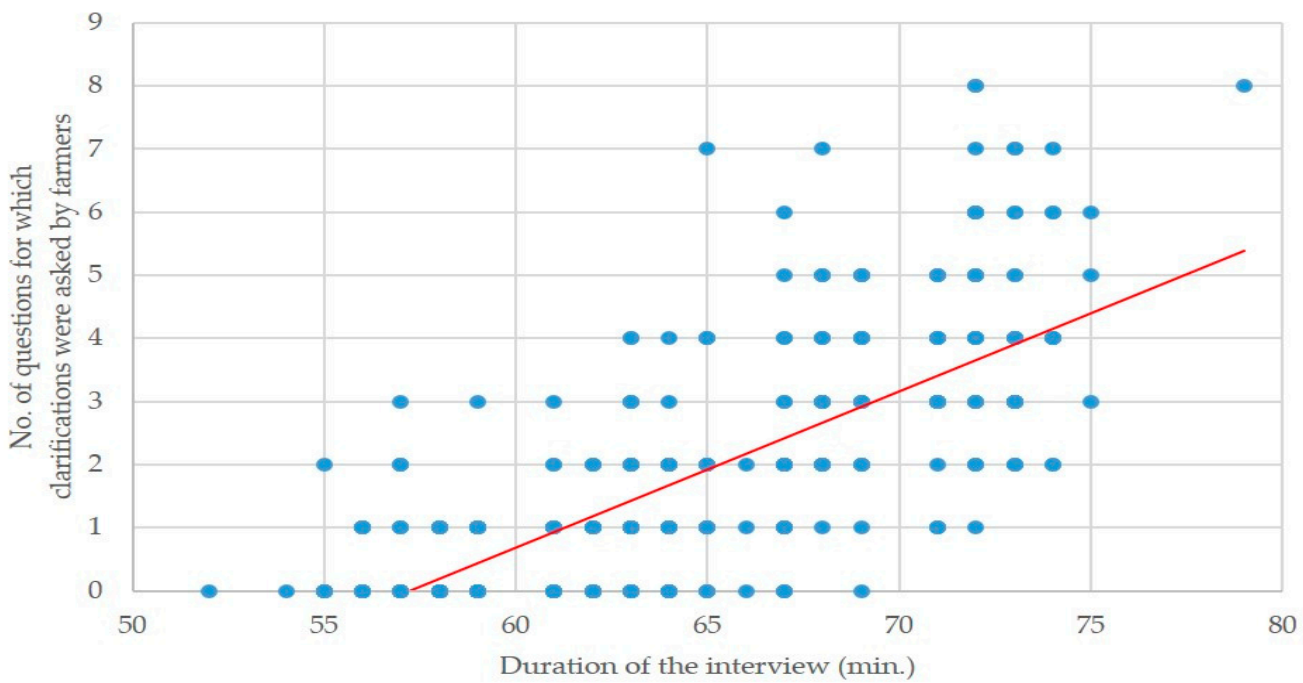

Figure 3. Plot diagram of the duration of the interview versus the number of clarifications asked by the farmers (solid red line indicates tendency line).

\subsubsection{Qualitative Assessment by Veterinarians and Farmers}

Of the 39 veterinarians from whom an opinion was acceptable (of the 47, two did not attend the interviews and six had been involved in the development of the questionnaire), 24 (61.5\%) spontaneously commented about the questionnaire and all $(100 \%)$ expressed a positive opinion (Table 3); of them, six (25.0\%) also requested a copy of the questionnaire to use in their practices. Among the 72 (16.4\% of all) farmers who spontaneously expressed an opinion, most $(n=51,70.8 \%)$ indicated that the questionnaire was lengthy and time-consuming; fewer farmers expressed a positive opinion ( $n=19,26.4 \%$; among them, three requested a copy of the questionnaire) or seemed annoyed with the many details asked $(n=2,2.8 \%)$; in those cases, farmers were reassured by the accompanying veterinarian that the interview was performed for research purposes only and not as an official evaluation of the farm.

Table 3. Frequency of the various qualitative comments about the questionnaire, spontaneously made by 24 veterinarians, who attended the interviews.

\begin{tabular}{cc}
\hline Comment & No. of Veterinarians Who Made the Comment \\
\hline Extensive and covering all relevant matters & 14 \\
Good & 12 \\
Useful for the small ruminant industry & 9 \\
Useful for practicing veterinarians & 6 \\
Very good & 6 \\
\hline
\end{tabular}

\subsubsection{Verification of Replies}

The replies of the farmers were verified against the details collected by the investigators during the site visit and the details obtained from the veterinarians, who had arranged the visits. In no case were discrepancies found between the replies of the farmers and the on-site observations of the investigators or the details provided by the veterinarians.

\section{Discussion}

The study has provided information regarding a detailed questionnaire to assess the health management in sheep flocks/goat herds in farms during a countrywide investigation in Greece. Questionnaires can provide valuable information for research and clinical purposes. To the best of our knowledge, the presented questionnaire is the most extensive and detailed one available for 
small ruminant farms in the international literature. The questionnaire is directed mainly for dairy farms, but, nevertheless, many of its questions can be easily applied during investigations in other production systems.

In previous reports, fewer questions were included in the relevant questionnaires, which were designed according to the specific needs of the research study performed. For example, in relation to work in sheep or goats, Lafi et al. [6] have used a 22-question document to study risk factors for $Q$ fever in Jordan; Muri et al. [7] have used a 30-question interview in Norway to study attitudes of sheep farmers; Morales-Pablos et al. [8] have used a 13-question document to evaluate risk factors for paratuberculosis in Mexico; Higino et al. [9] have used a 19-question document to assess risk factors for leptospirosis in Brazil; Delafosse et al. [10] have used a 35-question document to assess risk factors for cryptosporidiosis in France; Farkas and Hall [11] have used a 18-question document to study myiasis in Hungary. Use of questionnaires with small numbers of questions can lead in missing points and omitting collecting potentially valuable data during the field work, which would hinder correct analysis of the data at subsequent stages. Some of the questions asked in those questionnaires, have also been included in the present one (e.g., number and breed of animals in the farm; vaccination schedules, feeding regimes).

Use of similar questions in different research studies will also facilitate standardization of the work and easier comparison of the results between the studies. Questions from the present questionnaire can be combined with other ones, more specific and pertaining to the particular nature of a problem under study, which would be developed by researchers for specific projects. For example, in a field study regarding specifically reproductive management of sheep/goats, further relevant questions could be potentially added; examples include: in question 285, a description of the hormonal treatment (e.g., duration of intravaginal administration of progestogen sponges), and in question 290, the type of artificial insemination (e.g., intravaginal or intrauterine insemination) and the type of semen used (e.g., fresh or frozen semen). The above exemplify that specific additional questions can be formulated and added according to the particular needs of each occasion.

The present questionnaire can also be used as a model for recording farm details in cases of clinical work. Indeed, efficient history taking can be greatly facilitated by using a standardised questionnaire that could explore various issues and situations in the farm. The questionnaire can be handled by clinicians for collection of data in farms, in order to monitor changes in the situation with time. The usefulness of the questionnaire for such circumstances was corroborated by the unanimously positive comments of the clinicians and their request to have a copy for their own use in clinical work.

The recent establishment of EU-wide regulation regarding risk assessment in the food chain (which includes animal farms as the first step in the production of foods of animal origin) [12] underlines the usefulness of the present questionnaire, for collection and recording of detailed information in a structured way. Relevant national legislations also exist and the present questionnaire can also be adopted and applied in these approaches. For example, the 'ClassyFarm' is an Italian innovation and the result of a project commissioned by the Italian Ministry of Health, with the objective to improve and support the collaboration between farmers and the competent authorities of that country, in order to improve the safety and quality of products produced in animal farms. Under those circumstances, there is a potential to run the questionnaire to record and file detailed information in these farms. As part of assessments performed within the context of the above strategies, the questionnaire can be useful for recording in the field, storing the data and then evaluating the risk of the most frequent or probable problems in the participating farms.

Questionnaires with in-farm visits, such as the present investigation, have the advantage that they can provide an in-depth knowledge of the circumstances than telephone-, mail- or computer-based questionnaires [13]. This has become evident in the present work as farmers asked for clarifications during the interview, which would not have been possible if data had not been collected by personal visits. Further, a farm visit can be used also for collection of various samples, in order to obtain further data regarding the situation in a farm, depending on the specific purpose of a study. A significant 
disadvantage of in-farm surveys is the increased cost and time involved in collection of the data [13]; this can be frustrating in cases that the potential respondents refuse to accept the interview after arrival to the farm. The comments of the farmers indicated that a visit with a long questionnaire might not be always welcome, especially in periods of increased work-load at the farm (e.g., vaccination time, lambing season, harvesting period) [14].

The duration of the interview can be a factor that may adversely affect attitude of the farmers [15] and ultimately the quality of replies [16]. The duration of the interview can depend on the investigator and the respondent. The effect of the investigator was seen in the progressive reduction of the duration of the interviews. As the investigator presented the interview to more farmers, a small, but significant, reduction of the duration was evident. One may postulate that possibly, with time, the investigator could recite the questionnaire with less need to look into the questions, which ultimately contributed in the progressively reduced duration of the interviews.

Response latency, which is mirrored in the final duration of the interview, is the time necessary for a respondent to answer the questions and is considered to be associated with 'the amount of information processing necessary to answer a question' [17]. This was exemplified in the present study by farmers, who did not speak Greek as their primary language, taking significantly longer time to complete the interview. As these respondents did not ask for more clarifications than farmers who spoke Greek as their primary language, we postulate that the cause of the longer duration of the interview was possibly a latency in responding, rather than not fully understanding questions. Indeed, Wenz et al. [18] have reported that in face-to-face interviews respondents took more time to answer, but provided answers to more questions than in telephone-based interviews. Moreover, although it could be expected that older people would need more time to answer questions and that respondents with a higher educational level would be more familiar with answering questions, hence would need less time [19], no such effects were seen in this work. This can be the result of asking straightforward questions directly related to the daily professional circumstances and works of the respondents, coupled also with the fact that older farmers would be more experienced and proficient with professional issues, as well as more accustomed with management routine in their flocks/herds than younger, less experienced farmers.

The farmers were found to be positive and welcoming in the visits; the rate of refusals to an interview was negligible. Arrangement of the visit by the veterinarian, with whom the farmers were collaborating and whom they trusted, was a factor that obviously contributed. This also helped in practical ways, e.g., to arrange the visit at a time suitable for the farmer and to easily locate the farm at the countryside. A question that farmers often asked at the start of the visit was "How long will it take?"; this indicates the importance of the brevity of interviews for future survey work. The present results suggest that a 60- to 70-min-long interview would be near the longest period that farmers might accept.

Requests for clarifications by farmers were an important factor found to influence the duration of the questionnaire. Some of the points asked (e.g., embryo transfer, induction of lambing, colostrum bank) were the direct results of lack of relevant knowledge, as these are not applied frequently in small ruminant health management [20], hence farmers would not have heard of them at all.

During the initial assessment of the questionnaire, all farmers provided at least one inconsistent reply in the repeated interviews. Nevertheless, the high Cronbach' coefficient alpha indicated an excellent internal consistency of the replies to the questionnaire [21]. In most cases, the inconsistencies referred to open questions, which needed a description or the provision of qualitative data from the respondent. The frequent lack of detailed records regarding production characteristics in farms may contribute further to providing erroneous replies during the interviews. The findings indicate that answers to open-type questions, which seek descriptive information, should be treated more cautiously than answers to dichotomous or multiple-choice questions. In order to cross-check and verify as much as possible of the information obtained by farmers during the interviews, details were also recorded for some of the questions by other investigators during the on-site visit, whilst some other details were collected from the accompanying veterinarians. 
In the present study, the interviews were carried out consistently by the same investigator (DTL), who in fact performed the main investigation after an initial accustomisation and use of the questionnaire in farmers before the main study. This helped the investigator to familiarize themselves with the attitude of farmers and their way of responding to the questionnaire. Moreover, it minimized potential effects of the interviewer in the quality of data collected [22]. In cases of potential future use of the questionnaire in research work, it is, thus, recommended that initial small-scale field work is performed so that the future investigators familiarize themselves with the questionnaire and the approach of the farmers. As found in the present study, this might also help to decrease the duration of the interview. This, coupled with the performance of the interview by the same investigators, would contribute to increased data quality from the use of the questionnaire.

\section{Conclusions}

A detailed questionnaire to evaluate health management in small ruminant dairy farms has been developed, assessed and presented. The questionnaire includes 442 questions, arranged in seven sections. The questionnaire shows an excellent internal consistency as evidenced by a high Cronbach's coefficient alpha. The mean duration of the interview was $63.6 \mathrm{~min}$. Clarifications were often asked (by $61.5 \%$ of respondents) in $5.0 \%$ of the questions.

The questionnaire can be used for research work in the field, to record details in the farms under study. In accord with the needs of a particular study, it can be modified, by adding more specific questions or omitting others deemed to be less important. Moreover, it can also be used for routine monitoring purposes, as a useful means to record and maintain details of farms during clinical work. To the best of our knowledge, this is the most extensive and detailed relevant questionnaire available internationally for small ruminant farms.

Supplementary Materials: The following are available online at http://www.mdpi.com/2076-2615/10/9/1489/s1, Table S1: A detailed questionnaire for the evaluation of health management in sheep or goat dairy farms.

Author Contributions: Conceptualization: D.T.L., N.G.C.V. and G.C.F.; methodology: D.T.L., I.P.C., N.G.C.V., V.S.M., A.P.P., N.G.K., C.K.M., C.B., A.G., E.P., I.G., D.A.G. and A.I.K.; validation: D.T.L., I.P.C., C.K.M., G.C.F.; formal analysis: D.T.L. and I.P.C.; investigation: D.T.L., C.K.M., A.P.P., N.G.K, K.S.I. and G.C.F.; writing-original draft preparation: D.T.L. and G.C.F.; writing-review and editing: D.T.L., I.P.C., N.G.C.V., M.C., D.L. and G.C.F.; supervision: D.L., M.C., G.C.F.; project administration: G.C.F. All authors have read and agreed to the published version of the manuscript.

Funding: This research received no external funding.

Acknowledgments: The help received by E. Angelidou, E. Albanaki, M. Ali, C. Arsenopoulos, E. Charalambidis, M. Choutea, T. Chovolos, P. Dagkas, A. Daousi, V. Delistamatis, G. Dimou, I. Farmakis, E. Fotiadi, C. Gogos, A. Hantzara, T. Kanavos, T. Karatzinos, T. Kazakos, A. Koukoufikas, I. Lakafosi, G. Loufardaki, C. Mathiopoulos, I. Michalopoulos, G. Mousourakis, P. Ntais, D.C. Orfanou, S. Pakos, C. Pantopoulos, I. Papadakis, A. Papathanasiou, A. Papoutsi, C. Peleki, N. Petis, A. Pechlivanidis, P. Skourfounta, S.A. Spanos, M.C. Statira, E. Tassos, P. Toulatos, and S. Tsakalidis is greatly appreciated; these veterinarians contributed significantly by selecting farms and arranging the visits and the interviews, and also providing details about the flocks/herds.

Conflicts of Interest: The authors declare no conflict of interest.

\section{Appendix A}

Questions for which relevant details were recorded (additionally to the answers received during the interview) during a thorough on-site evaluation of all relevant areas in the farm during 444 visits. 


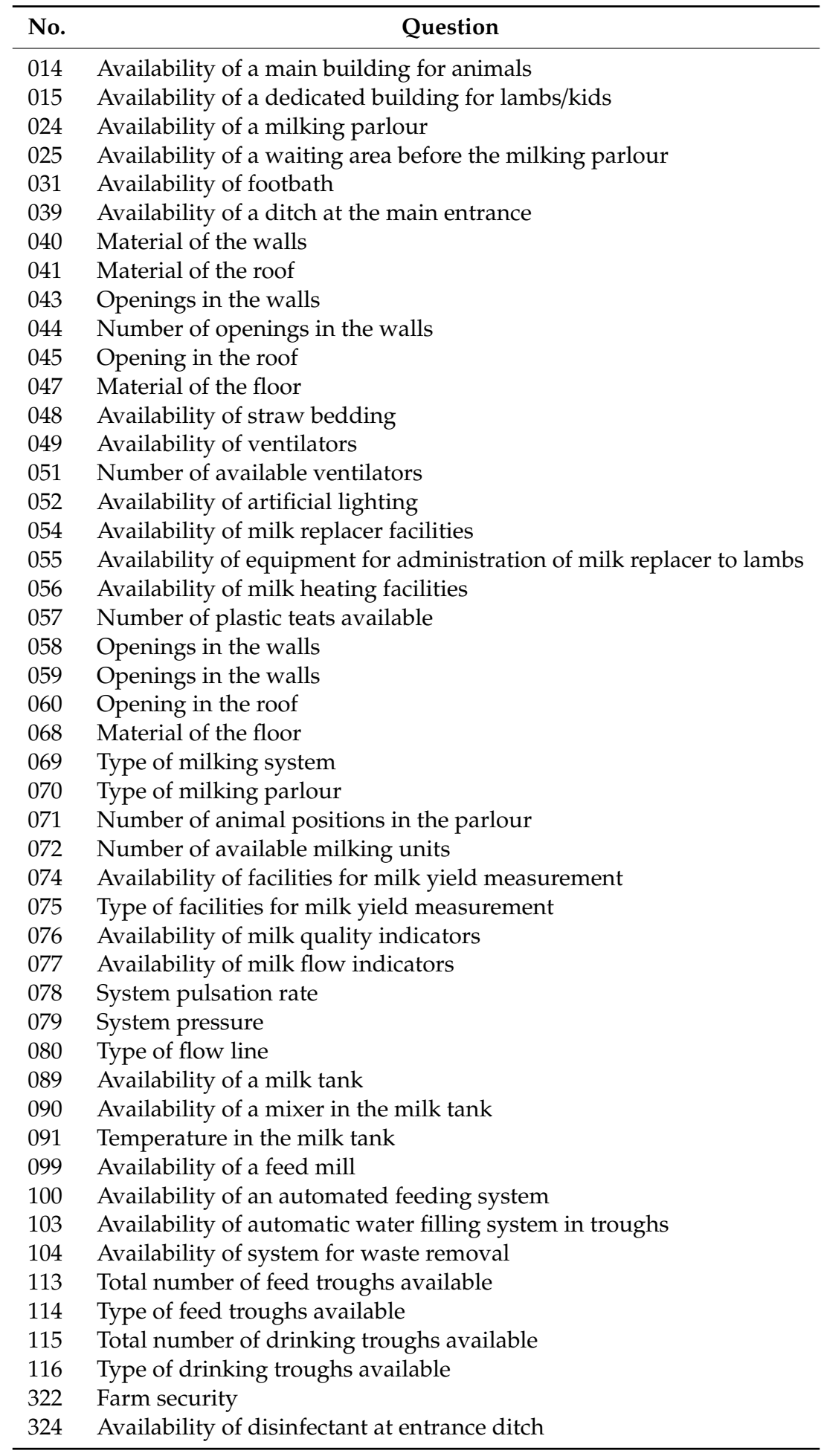

\section{Appendix B}

Sheep flocks or goat herds visited in the 13 administrative regions of Greece, for completion of the questionnaire. 


\begin{tabular}{lcc}
\hline Administrative Region & No. of Sheep Flocks & No. of Goat Herds \\
\hline Attica & 1 & 1 \\
Central Greece & 26 & 6 \\
Central Macedonia & 25 & 11 \\
Crete & 19 & 10 \\
Eastern Macedonia and Thrace & 34 & 16 \\
Epirus & 18 & 4 \\
Ionian islands & 3 & 1 \\
North Aegean & 19 & 3 \\
Peloponnese & 45 & 25 \\
South Aegean & 2 & 2 \\
Thessaly & 71 & 18 \\
Western Greece & 45 & 17 \\
Western Macedonia & 17 & 5 \\
Total & 325 & 119 \\
\hline
\end{tabular}

\section{Appendix C}

Questions for which relevant details were collected (additionally to the answers received during the interview) from the veterinarians, who had arranged the visits and had an association with the respective farms, during 444 visits.

\begin{tabular}{|c|c|}
\hline No. & Question \\
\hline 260 & Diseases of adult animals-mastitis: Total cases during the preceding season \\
\hline 261 & Diseases of adult animals-mastitis: Sample collection and testing for diagnostic purposes \\
\hline 262 & Diseases of adult animals—mastitis: Treatment \\
\hline 263 & Diseases of adult animals-mastitis: Pharmaceuticals used for treatment \\
\hline 264 & Diseases of adult animals_-mastitis: Route for administration of antimicrobials \\
\hline 265 & Diseases of adult animals-abortion: Total cases during the preceding season \\
\hline 266 & Diseases of adult animals_-abortion: Sample collection and testing for diagnostic purposes \\
\hline 267 & Diseases of adult animals—abortion: Types of samples collected for testing \\
\hline 268 & Diseases of adult animals-abortion: Pharmaceuticals used for treatment \\
\hline 269 & Diseases of adult animals-abortion: Collection of aborted material for safe disposal \\
\hline 270 & Diseases of adult animals_-pregnancy toxaemia: Total cases during the preceding season \\
\hline 271 & Diseases of adult animals_-pregnancy toxaemia: Treatment performed \\
\hline 272 & Diseases of adult animals_-lameness: Total cases during the preceding season \\
\hline 273 & Diseases of adult animals-lameness: Treatment performed \\
\hline 274 & Diseases of adult animals-mange: Total cases During the preceding season \\
\hline 275 & Diseases of adult animals_-mange: Treatment performed \\
\hline 276 & Diseases of adult animals-obstetrical cases: Total cases during the preceding season \\
\hline 277 & Diseases of adult animals_-obstetrical cases: Call for veterinary support \\
\hline 279 & Diseases of young animals_-respiratory problems: Total cases during the preceding season \\
\hline 280 & Diseases of young animals-respiratory problems: Treatment performed \\
\hline 281 & Diseases of young animals_-respiratory problems: Pharmaceuticals used for treatment \\
\hline 282 & Diseases of young animals-diarrhoea: Total cases during the preceding season \\
\hline 283 & Diseases of young animals_-diarrhoea: Treatment performed \\
\hline 284 & Diseases of young animals-diarrhoea: Pharmaceuticals used for treatment \\
\hline 325 & Vaccinations: Against Chlamydia infection \\
\hline 326 & Description of schedule: \\
\hline 327 & Vaccinations: Against Toxoplasma infection \\
\hline 328 & Description of schedule: \\
\hline 329 & Vaccinations: Against Brucella infection \\
\hline 330 & Description of schedule: \\
\hline 331 & Vaccinations: Against clostridial infection \\
\hline 332 & Description of schedule: \\
\hline 333 & Vaccinations: Against mastitis \\
\hline 334 & Description of schedule: \\
\hline 335 & Vaccinations: Against contagious agalactia \\
\hline 336 & Description of schedule: \\
\hline 337 & Vaccinations: Against bacterial respiratory infections \\
\hline
\end{tabular}




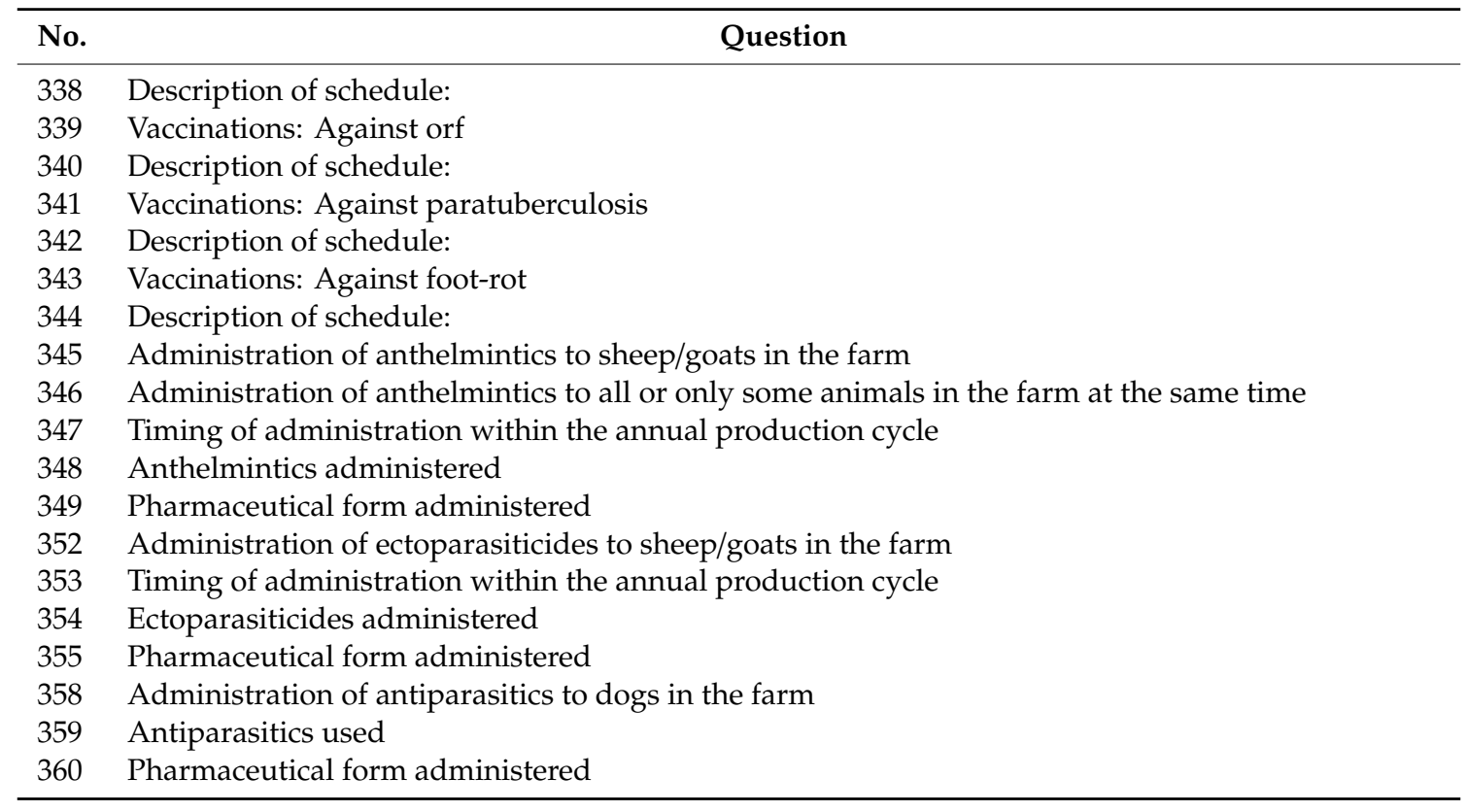

\section{Appendix D}

Questions in which inconsistencies were recorded in the answers of 27 farmers, interviewed by two different persons two to three months apart.

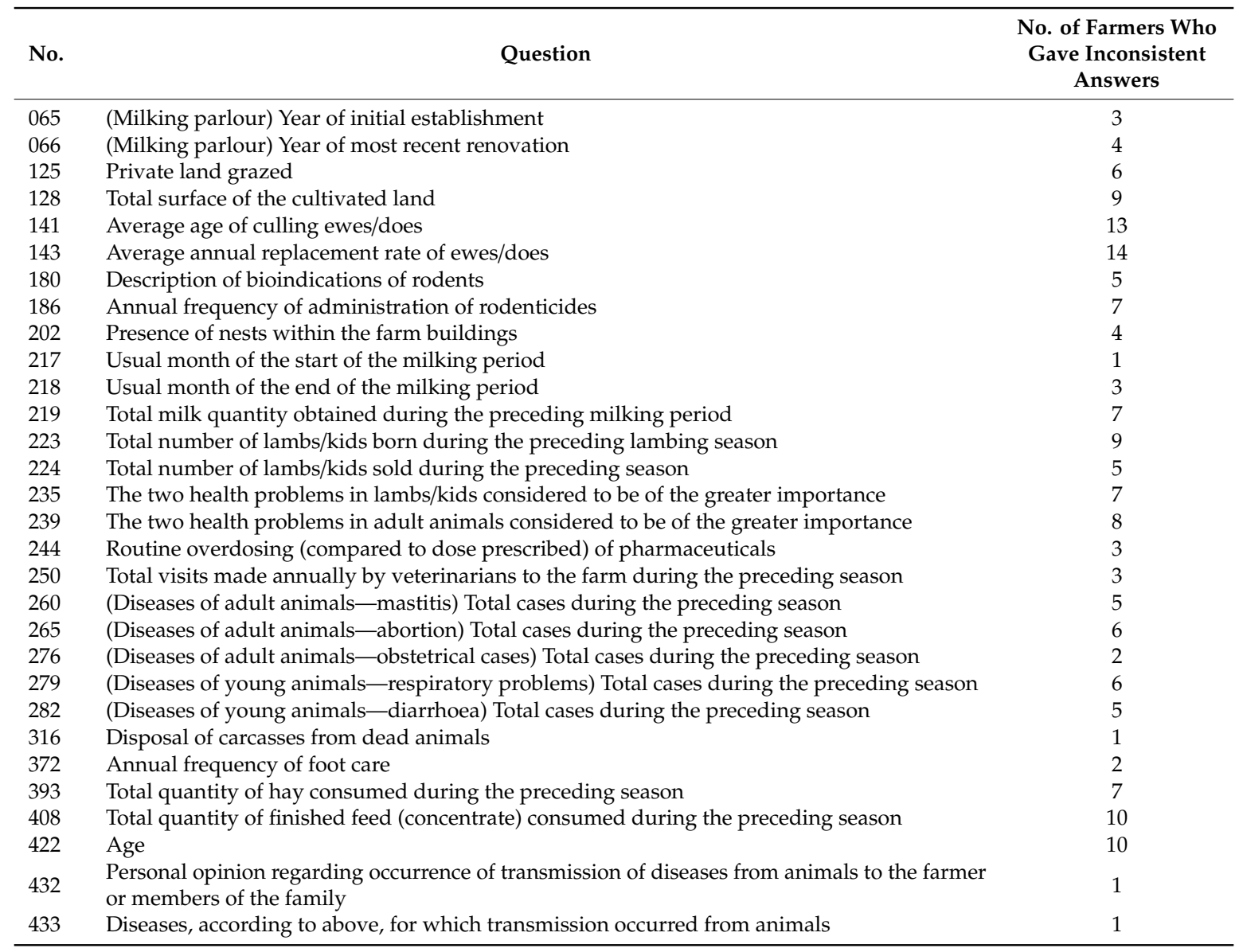




\section{Appendix E}

Questions in which clarifications were asked by farmers during 444 interviews.

\begin{tabular}{llc}
\hline No. & \multicolumn{1}{c}{ Question } & $\begin{array}{c}\text { No. of Farmers who Requested } \\
\text { Further Explanations }\end{array}$ \\
\hline 016 & Availability of a dedicated lambing/kidding area & 14 \\
035 & Proximity to industrial sites & 7 \\
076 & Availability of milk quality indicators & 38 \\
077 & Availability of milk flow indicators & 7 \\
080 & Type of flow line & 30 \\
083 & Type of system check-ups performed by technicians & 19 \\
098 & Availability of a roller crusher & 9 \\
103 & Availability of automatic water filling system in troughs & 10 \\
112 & Availability of animal location identifiers & 23 \\
119 & Availability of sensors for registration of environmental conditions & 24 \\
141 & Average age of culling ewes/does & 76 \\
146 & Criteria for selection of own animals as replacements & 47 \\
245 & Use of laboratory diagnostic examinations & 61 \\
253 & Evaluation of ammonia concentration within the buildings & 36 \\
287 & Changes of rams/bucks into the ewes/does during the mating period & 63 \\
288 & Castration of lambs/kids kept for fattening & 5 \\
289 & Use of vasectomies & 56 \\
291 & Use of embryo transfer & 89 \\
293 & Nutritional modifications before the mating period & 12 \\
300 & Induction of lambing & 38 \\
303 & Lamb/kid fostering to female animals other than their dams & 5 \\
394 & Plants included in hay consumed by animals & 46 \\
\hline
\end{tabular}

\section{References}

1. Federation of Veterinarians of Europe. Veterinary Act-European Veterinary Code of Conduct; Federation of Veterinarians in Europe: Brussels, Belgium, 2009.

2. Hellenic Veterinary Association. Code of Correct Veterinary Practice; Hellenic Veterinary Association: Athens, Greece, 2011.

3. European Food Safety Authority. Scientific opinion on the welfare risks related to the farming of sheep for wool, meat and milk production. EFSA J. 2014, 12, 3933-4060.

4. Cronbach, L.J. Coefficient Alpha and the internal structure of tests. Psychometrika 1951, 16, 297-334.

5. Wessa, P. Free Statistics Software, Office for Research Development and Education. Available online: https://www.wessa.net/ (accessed on 29 July 2020).

6. Lafi, S.Q.; Talafha, A.Q.; Abu-Dalbouh, M.A.; Hailat, R.S.; Khalifeh, M.S. Seroprevalence and associated risk factors of Coxiella burnetii (Q fever) in goats and sheep in northern Jordan. Trop. Anim. Health Prod. 2020, 52, 1553-1559. [CrossRef]

7. Muri, K.; Tufte, P.A.; Coleman, G.; Moe, R.O. Exploring work-related characteristics as predictors of Norwegian sheep farmers' affective job satisfaction. Sociol. Rural. 2020, 60, 574-595. [CrossRef]

8. Morales-Pablos, M.I.; Mejia-Sanchez, P.; Diaz-Aparicio, E.; Palomares-Resendiz, E.G.; Gutierrez-Hernandez, J.L.; Reyna-Granados, J.R.; Luna-Nevarez, P.; Munguia-Xochihua, J.A.; Segura-Correa, J.C.; Leyva-Corona, J.C. Risk factors associated with the seroprevalence of paratuberculosis in sheep flocks in the hot-arid region of Sonora, Mexico. Trop. Anim. Health Prod. 2020, 52, 1357-1363. [CrossRef]

9. Higino, S.S.S.; Santos, F.A.; Costa, D.F.; Santos, C.S.A.B.; Silva, M.L.C.R.; Alves, C.J.; Azevedo, S.S. Flock-level risk factors associated with leptospirosis in dairy goats in a semiarid region of Northeastern Brazil. Prev. Vet. Med. 2020, 109, 158-161. [CrossRef]

10. Delafosse, A.; Castro-Hermida, J.A.; Baudry, C.; Ares-Mazas, E.; Chartier, C. Herd-level risk factors for Cryptosporidium infection in dairy-goat kids in western France. Prev. Vet. Med. 2006, 77, 109-121. [CrossRef]

11. Farkas, R.; Hall, M.J.R. Prevalence of traumatic myiasis in Hungary: A questionnaire survey of veterinarians. Vet. Rec. 1998, 143, 440-443. [CrossRef] 
12. European Union. Regulation (EU) 2019/1381 of the European Parliament and of the Council of 20 June 2019 on the transparency and sustainability of the EU risk assessment in the food chain and amending Regulations (EC) No 178/2002, (EC) No 1829/2003, (EC) No 1831/2003, (EC) No 2065/2003, (EC) No 1935/2004, (EC) No 1331/2008, (EC) No 1107/2009, (EU) 2015/2283 and Directive 2001/18/EC. Off. J. Eur. Union 2019, L231, 1-28.

13. Crawford, I.M. Agricultural and Food Marketing Management; FAO Regional Office for Africa: Harare, Zimbabwe, 1990.

14. Crawford, I.M. Marketing Research and Information Systems; Food and Agriculture Organisation: Rome, Italy, 1997.

15. Galesic, M.; Bosnjak, M. Effects of questionnaire length on participation and indicators of response quality in a web survey. Public Opin. Q. 2009, 73, 349-360. [CrossRef]

16. Bogen, K. The effect of questionnaire length on response rates-A review of the literature. In Census Authors' Papers; U.S. Bureau of the Census: Suitland, MD, USA, 1996.

17. Bassili, J.N.; Scott, B.S. Response latency as a signal to question problems in survey research. Public Opin. Q. 1996, 60, 390-399. [CrossRef]

18. Wenz, A.; Al Baghal, T.; Gaia, A. Language proficiency among respondents: Implications for data quality in a longitudinal face-to-face survey. J. Surv. Stat. Methodol. 2020, 8. [CrossRef]

19. Loosveldt, G.; Beullens, K. 'How long will it take?' An analysis of interview length in the fifth round of the European Social Survey. Surv. Res. Methods 2013, 7, 69-78. [CrossRef]

20. Sargison, N. Sheep Flock Health a Planned Approach; Blackwell Publishing: Oxford, UK, 2008.

21. Glen, S. Statistics for the Rest of Us; Statistics How To: Jacksonville, FL, USA, 2014.

22. Davis, R.E.; Couper, M.P.; Janz, N.K.; Caldwell, C.H.; Resnicow, K. Interviewer effects in public health surveys. Health Educ. Res. 2010, 25, 14-26. [CrossRef]

(C) 2020 by the authors. Licensee MDPI, Basel, Switzerland. This article is an open access article distributed under the terms and conditions of the Creative Commons Attribution (CC BY) license (http://creativecommons.org/licenses/by/4.0/). 